\title{
Clinical comparison of induction with inhalational sevoflurane versus intravenous propofol for perioperative anesthetics in adult: a randomized clinical trial
}

Background: The introduction of target controlled infusion with propofol and the use of vital capacity inhalation with sevoflurane has led to the rediscovery of 'optimal' induction conditions.

Objective: To compare sevoflurane and propofol as induction agents, focusing on their impact on haemodynamic stability and anesthetic consumption used during anesthesia maintenance as well as recovery characteristics.

Methods: Sixty patients of physical status according to the American Society of Anesthesiologist (ASA) scale of I-II, (2565 years) undergoing selective lumbar spondylodesis were randomized into two anesthesia induction groups: target control, propofol infusion or vital capacity inhalation with $8 \%$ sevoflurane. Patients subsequently underwent muscular paralysis and intubation, followed by anesthesia maintenance with propofol/remifentanil.

Main outcome measure: An independent observer recorded the time to the target depth of anesthesia using the Narcotrend index, hemodynamic stability, and overall anesthetic consumption.

Results: Hemodynamic stability was maintained and comparable between both groups. A vital capacity inhalation with $8 \%$ sevoflurane resulted in a significantly shorter induction time to target anesthesia depth than the target controlled infusion with propofol but was associated with a higher incidence of hypoventilation. The amount of propofol/ remifentanil needed for anesthesia maintenance was substantially less in patients induced with sevoflurane compared to those induced with propofol.

Conclusion: The vital capacity inhalation with $8 \%$ sevoflurane was comparable to target control infusion with propofol with respect to the speed of induction, smooth anesthesia depth, and amount of anesthetic consumption during maintenance.

KEYWORDS: anesthesia induction, vital capacity inhalation with sevoflurane, target controlled infusion with propofol, anesthesia maintenance, narcotrend index

\section{Abbreviations}

VCI: Vital Capacity Inhalation; VCI-S: Vital Capacity Inhalation with Sevoflurane; TCI: Target Control Infusion

TCI-P: Target Control Infusion with Propofol; Ce: Effect-site Concentration; Cp: Plasma Concentration; NI: Narcotrend Index; NTS: Narcotrend Stage; ASA:A Physical Status with American Society of Anesthesiologist; ECG: Electrocardiography; BP: Noninvasive Arterial Blood Pressure; SP: Systolic Pressure; DP: Diastolic Pressure;
HR: Heart Rate; $\mathrm{SpO}_{2}$ : Peripheral Oxygen Saturation; $\mathrm{P}_{\mathrm{ET}} \mathrm{CO}_{2}$ : End-Tidal Carbon Dioxide Partial Pressure; IP: Mean Inspiratory Pressure; ETS evo: End-Tidal Concentration of the Volatile Anesthetic Sevoflurane; MAC: Minimum Alveolar anesthetic Concentration; PACU: Post-Anesthesia Care Unit; EEG: Electroencephalography; USB: Universal Serial Bus; ECT: Electroconvulsive Therapy

\section{Impacts on practice}

1. On approaching patients preoperatively, inhalational induction with sevoflurane
Chunshan Dong*, Peng Sun, Junma Yu, Jun Zhang \& Chao Wu Third affiliated hospital of An Hui Medical University, HeFei, AnHui China *Author for correspondence: cxh0909@vip.126.com 
was not as popular as the use of intravenous induction agents in adult, but it as an induction techniques that have been introduced the past ten years and have proved some benefit from its faster induction and emergence, which could mean adjustments in anesthetics practice can be more flexible.

2. There have been most studies comparing propofol with sevoflurane for induction, maintenance, and recovery, but not several clinical trials study the influence of anesthetic induction on anesthetics maintenance. Nevertheless, our study provided useful information.

3. An inhalational technique with sevoflurane seem a most cost-effective option, on one hand, it decreases the consumption of anesthetic agents during maintenance. On the other hand, it may decreases direct costs of anesthesia due to the price of the special electrodes used by intravenous infusion.

\section{Introduction}

Safe, effective, general anesthestic protocols have developed in tandem with increasingly complex operations and procedures. The challenge for the anesthesiologist is to provide optimal surgical conditions while, at the same time, ensuring adequate oxygenation to the brain and other organs. A wide variety of standard induction techniques and maintenance regimens have been used, mostly with acceptable results. Combinations of anesthetic medications that produce similar effects and act their similar or different mechanisms are commonly used, resulting in pharmacodynamically- significant interactions between them. These interactions are varied, too, in part, because of the chemical heterogeneity of the compounds and their action through different mechanisms. There are three types of pharmacodynamics interactions: synergistic, additive, or infra-additive (or when the combined effect of both drugs is greater, equal, or less than, respectively, the sum of the effects of either drug alone) [1]. Synergistic interactions can be useful, in particular, because they allow for lower doses of each drug to be used, thereby reducing the possibility of side effects.

A common strategy used in routine clinical practice is to perform the induction with an intravenous formulation (such as propofol) and to continue maintenance with an inhaled agent (such as sevoflurane). While numerous studies have been published comparing inhaled versus intravenous agents for the induction and maintenance of anesthesia in several surgical procedures, study protocols vary with regard to pre-medication, inhalational agents, fresh gas flow rates, additional opioids usage, and the type and duration of surgery [2-4]. Furthermore, less attention has been paid to assess whether there may be varying 'legacy' effects of anesthesia induction caused by the different anesthetic techniques through variable factors such as the induction speed or the induction quality (or both). Moreover, few studies have compared the differential effects of sevoflurane versus proprofol induction on hemodynamic stability, dosage consumption, and adverse effects during maintenance with propofol/ remifentanil.

Previous studies have demonstrated that general anesthesia can be induced in patients by allowing them to inhale sevoflurane (a sweet-smelling inhaled anesthetic drug) combined with oxygen through a mask [5]. This technique has been reported to be safe, reliable, and well-accepted and tolerated by patients. Based on these observations, we hypothesized that combining high concentration sevoflurane-based vital capacity inhalation (VCI) with remifentanil, a short-acting opioid would provide a better haemodynamic profile and more reasonable anesthetic regimen for the rapid induction of anesthesia. In this study, we compared anesthesia with VCI inhalational sevoflurane combined with target-controlled infusion (TCI) remifentanil versus TCI propofol combined with TCI remifentanil, followed by TCI with both propofol and remifentanil for anesthesia maintenance in adult patients undergoing lumbar spondylodesis under Narcotrend Index (NI) or Narcotrend stage (NTS) monitor.

\section{Aim of the study}

The primary target variable of this study was to compare two standard induction techniques using VCI of sevoflurane and TCI of propofol by monitoring NI or NTS. The null hypothesis was that the perioperative anesthetics were similar with two induction techniques in adult, and the alternative hypothesis was that they were different. The primary outcome variable was whether there were significantly different with two induction techniques, which was shown to be any potential benefits for the quality of induction and recovery characteristics, and resulted in a significantly haemodynamic stabilityas and reduced requirement of drugs 
during anesthesia maintenance for patients undergoing spine surgery.

\section{Ethical approval}

This prospective, randomised clinical trial was approved by the Research and Ethics Committee of the Affiliated Third Hospital of AnHui medical university (HeFei, China). From January 2013 to January 2015.

\section{Method}

Patients aged 25-65, with a physical status according to the American Society of Anesthesiologist (ASA) scale of I-II, and undergoing selective lumbar spondylodesis were prospectively enrolled in the study. Informed consent was obtained from all patients. Exclusion criteria included if patients were unable to cooperate, had a history of sedative drugs use, mental disorders, malignant hyperthermia, respiratory disease, adverse reaction to sevoflurane or propofol, as well as severe cardiovascular, neurologic, hepatic, or renal disease. After obtaining the informed consent, patients were allocated randomly to their PCIA regimen using a computer-generated random table (using simple randomization method) into two groups. Group assignments were placed inside numbered opaque enveloped as follows: VCI of sevoflurane (group VCI-S) and TCI of propofol (group TCI-P). The vital capacity technique was explained to patients in the anesthetic induction room, and all patients were not pre-medicated.

Before induction with anesthesia, each patient was monitored via electrocardiography (ECG), noninvasive arterial blood pressure (BP), heart rate (HR), and peripheral oxygen saturation $\left(\mathrm{SpO}_{2}\right)$. An NTS and NI sensor was attached to each patient in conjunction with the Narcotrend monitor (MonitorTechnik, Bad Bramstedt, Germany). Peripheral IV access was established with a 20G cannula and a crystalloid infusion was started at $5 \mathrm{ml} / \mathrm{kg} / \mathrm{h}$. In the VCI-S group, each patient was instructed to inhale as deeply as possible and subsequently to exhale to residual volume. The anesthesia circuit was primed with a reservoir bag filled with $8 \%$ sevoflurane (ShangHai hengrui pharmaceutical $\mathrm{CO}$, China) and $3 \mathrm{~L} /$ minute fresh oxygen flow (Datex-Ohmeda Aestive/5 Smart Ventilator, Madison, WI) for $1 \mathrm{~min}$. Patients were informed told about the odor of the volatile gas prior to anesthesia induction. During induction, the patient inspired $8 \%$ sevoflurane with a fresh gas flow of $6 \mathrm{~L} / \mathrm{min}$ pure oxygen via a sealed face mask combined with a target-controlled infusion (TCI) (pump using SIMS Graseby Ltd, Watford, UK. Diprifusor Software; Aspect Medical, Inc., Newton, Massachusetts, USA) of remifentanil (YiChang people CO, China). The the initial effect-site concentration (Ce) target was $11 \mathrm{ng} / \mathrm{ml}$, which was reduced to $6 \mathrm{ng} / \mathrm{ml}$, after 1 minute [6]. Patients were encouraged to take additional deep breaths until they were asleep. Loss of consciousness (LOC) was assessed repeatedly by loss of response to command and loss of lash reflex. After LOC, the svoflurane vaporizer was set to $3 \%$ sevoflurane with a fresh gas flow of $3 \mathrm{~L} / \mathrm{min}$ pure oxygen via a sealed face mask. In the TCI-P group, prior to anesthetia induction, all patients were pre-oxygenated with $3 \mathrm{~L} / \mathrm{min}$ pure oxygen via a sealed face mask. The patient's age and weight was entered into the TCI (the same pump was used as for VCI-S group) unit enabling so target propofol (Diprivan, AstraZeneca, UK) and remifentanil concentration to be set and the infusion started, and the initial target induction plasma concentration (Cp) of propofol was $6.5 \mu \mathrm{g} /$ $\mathrm{ml}$, which was reduced to $3 \mu \mathrm{g} / \mathrm{ml}$ after LOC, combined with TCI of remifentanil (effectsite concentration same with group VCI-S). In addition, the patient inhaled a fresh gas flow of $3 \mathrm{~L} / \mathrm{min}$ pure oxygen via a sealed face mask. Patients in both groups were asked to open their eyes every $10 \mathrm{~s}$ during induction. Loss of response to open the eyes and loss of response to command were interpreted as LOC. All patients received cisatracurium (ShangHai hengrui pharmaceutical CO, China) $0.2 \mathrm{mg} / \mathrm{kg}$ titrated after LOC to ensure muscular paralysis until NTS stages reached the level $E_{0}-E_{1}$, after which the tracheal intubation was performed. All drugs were discontinued after NTS stages reached the level $\mathrm{E}_{0}-\mathrm{E}_{1}$, and after intubation, all patients were received the same anesthesia maintenance with a variable concentration infusion regimen of propofol and remifentanil, during TCI, the highest concentration $(\mathrm{Cp})$ of propofol was 8 $\mu \mathrm{g} / \mathrm{ml}$, the lowest concentration of propofol was $3 \mu \mathrm{g} / \mathrm{ml}$; and the highest concentration (Ce) of remifentanil was $6 \mathrm{ng} / \mathrm{ml}$, the lowest concentration of remifentanill was $2 \mathrm{ng} / \mathrm{ml}$. A same step increasing or decreasing methods for propofol and remifentanil were adjusted as necessary to maintain an NTS levels between grade $\mathrm{D}_{2}$ and grade $\mathrm{E}_{0}$ as well as haemodynamic responses to surgical stimuli. Following intubation, the end-tidal carbon dioxide partial pressure $\left(\mathrm{P}_{\mathrm{ET}} \mathrm{CO}_{2}\right)$ was measured using an infrared analyzer $\left(\mathrm{D}\right.$-fand ${ }^{\mathrm{TM}}$, E-CAiO-OO, GE 
Healthcare Finland Oy Helsinki, Finland) and ventilation was adjusted to maintain $\mathrm{P}_{\mathrm{ET}} \mathrm{CO}_{2}$ values between $35-45 \mathrm{mmHg}$. Cisatracurium boluses were administered at $0.06 \mathrm{mg} / \mathrm{kg}$ if required and fluid administration was adjusted as needed throughout the duration of anesthesia maintenance to maintain hemodynamic stability in all patients. Cisatracurium was discontinued 30 minute prior to the completion of the procedure as were other procedure-related drugs as appropriate. All patients were observed in the post-anesthesia care unit (PACU) for at least 1 hour, where standard monitoring was administered.

Depth of anesthesia was quantified using EEG analysis that was connected to the Narcotrend monitor. Appropriate monitoring equipment was placed on each patient prior to anesthesia induction, and electrode positioning was optimized until the impedances were $<5 \Omega$. The Narcotrend algorithm integrates stages to a numerical scale from 100 to 0 , assigning a defined range of index values to each of the EEG stages: A (awake) to F (NTS). A multivariate classification function associates an EEG epoch to an index value between 100 and 0 (NI). The values generated by the monitor were recorded at 5-20 seconds intervals on a USB memory stick. The Narcotrend was used to guide the depth of anesthesia, and NTS and NI recordings were started $10 \mathrm{~min}$ before induction of anesthesia and continuously performed until the patients fulfilled the clinical criteria after extubation at 10 minutes.

In all the patients, HR, systolic pressure (SP), diastolic pressure (DP), $\mathrm{SpO}_{2}$, and NI were recorded before the anesthesia induction (baseline, $\mathrm{T}_{0}$ ). HR, SP, DP, $\mathrm{SpO}_{2}$, and NI were recorded at the time points as follows: laryngoscopy $\left(\mathrm{T}_{1}\right)$ when NTS level decreased to $\mathrm{E}$ stage; after a tracheal intubation at $5 \mathrm{~min}$ $\left(\mathrm{T}_{2}\right)$, and $10 \mathrm{~min}\left(\mathrm{~T}_{3}\right)$; after patient was turned to the prone position at $5 \mathrm{~min}\left(\mathrm{~T}_{4}\right)$ and during the procedure at $10 \mathrm{~min}\left(\mathrm{~T}_{5}\right)$; patient was returned to the supine position after the end of procedure $\left(\mathrm{T}_{6}\right)$ and during the extubation $\left(\mathrm{T}_{7}\right)$; resumption of spontaneous breathing after the end of procedure $\left(\mathrm{T}_{8}\right)$ and before the patient was transferred to the PACU $\left(\mathrm{T}_{9}\right)$. All adverse effects associated with induction were recorded, such as coughing, breath holding, secretions, excitatory movements, and laryngospasm. The times from the onset of induction of anesthesia until the NST stage decreases to $\mathrm{D}_{2}$ and $\mathrm{E}_{0}$ as well as the duration of anesthesia and surgery were also recorded. After completion of surgery, the dosage of remifentanil and propofol used for anesthesia maintenance (not including the dose used for induction) were calculated based on the volumes of anesthetics that were used during TCI as calculated by ascertaining the amount left on the syringe driver at appropriate elapsed times during the protocol. Dose of cisatracurium administered was calculated based on the actual consumption per operation. Upon transfer to the PACU, patients were evaluated for the resumption of spontaneous breathing, response to verbal instruction, orientation (in place and time, which provides a rough estimation of the recovery of cognitive function), and extubation, as well as the time from the completion of procedure to the return of cognitive function. Adverse effects such as excitatory agitation, nausea, and vomiting during this period were also recorded. Moreover, 2hours after complete recovery from their anesthetic, patients were asked to subjectively assess the acceptability of their induction and whether they would choose the same type of induction again. Their responses were recorded as "pleasant", neither pleasant nor unpleasant," or "unpleasant".

\section{Data analysis}

Statistical analysis was performed using SPSS (Chicago, IL) version 15 for Windows. Values are expressed as mean \pm SD. Continuous variables were analyzed using a two-tailed unpaired student's t test or ANOVA when appropriate. For categorical variables (i.e. adverse events), $\chi^{2}$ tests were used. Independent sample t-test or the nonparametric equivalent Mann-Whitney test or ANOVA for repeated measures with post boc analysis were also conducted to evaluate for group differences at each time point. The significance for such differences was adjusted using the Bonferroni correction. P-value $<0.05$ was considered statistically significant.

\section{Results}

\section{- Patient's characteristics}

The CONSORT patient flow diagram is shown in FIGURE 1. A total of sixty patients were enrolled in this study and randomized to either the propofol intravenous infusion group or the sevoflurane inhalation group $(n=30$ per group). The patients within each group were comparable with respect to demographic data as well as mean duration of surgery and anesthetic time (TABLE 1). 


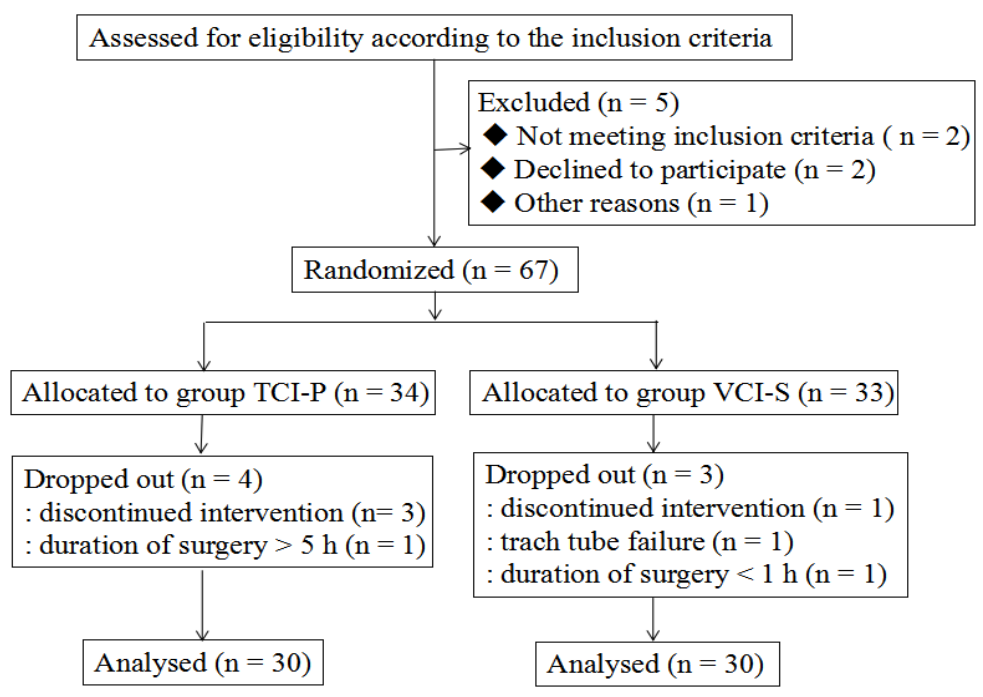

Patients enrolment flow diagram.

FIGURE 1. Patients enrollment flow diagram. TCI-P, target-controlled infusion with propofol; VCI-S, vital capacity inhalation with sevoflurane.

Table 1. Patients characteristics duration of anesthetic and surgery. Values are mean \pm SD [95\% $\mathrm{Cl}$. No differences between the groups were detected.

\begin{tabular}{|c|c|c|c|c|}
\hline & $\begin{array}{c}\text { Group TCI-P } \\
(\mathrm{n}=30)\end{array}$ & $\begin{array}{c}\text { Group VCI-S } \\
(\mathrm{n}=30)\end{array}$ & $\mathrm{T}$ \\
\hline Age $(\mathrm{yr})$ & $48.3 \pm 14.0$ & $49.1 \pm 11.3$ & -0.243 & 0.809 \\
\hline Gender (M/F) & $11 / 19$ & $12 / 18$ & \\
\hline ASA (I/II) & $22 / 8$ & $20 / 10$ & \\
\hline Weight (kg) & $60.7 \pm 9.6$ & $64.1 \pm 10.9$ & -1.259 & 0.213 \\
\hline Height (cm) & $166.3 \pm 9.5$ & $164.9 \pm 9.5$ & 0.558 & 0.579 \\
\hline $\begin{array}{c}\text { Duration of anesthetic } \\
\text { (min) }\end{array}$ & $183.3 \pm 32.6$ & $185.9 \pm 32.3$ & -0.302 & 0.763 \\
\hline $\begin{array}{c}\text { Duration of surgery } \\
\text { (min) }\end{array}$ & $166.5 \pm 33.7$ & $170.4 \pm 34.5$ & -0.447 & 0.657 \\
\hline
\end{tabular}

SD: standard deviation, Cl: confidence limits, M/F: male/female, ASA: American Society of Anesthesiologists' physical status

\section{Comparison of NI during anesthetics induction}

The defined NTS target level $\left(\mathrm{E}_{0}-\mathrm{E}_{1}\right)$ during tracheal intubation and the target level $\left(\mathrm{D}_{2} \sim \mathrm{E}_{0}\right)$ during anesthesia maintenance were achieved in both groups. The mean NI values were significantly higher in the TCI-P group than that in the VCI-S group after tracheal intubation at 5 minutes $\left(\mathrm{T}_{2}: 43.3 \pm 3.9\right.$ versus $36.3 \pm 4.7$, respectively [please verify]). But the mean NI values were significantly lower in the TCI-P group than that in the VCI-S group from $\mathrm{T}_{7}$ to $\mathrm{T}_{9}\left(\mathrm{~T}_{7}: 75.8 \pm 4.4\right.$ versus $80.5 \pm$ 5.2 , respectively; $\mathrm{T}_{8}: 83.1 \pm 4.4$ versus 85.6 \pm 3.3 ; and $\mathrm{T}_{9}: 88.5 \pm 3.9$ versus $91.8 \pm 3.3$ ) (FIGURE 2).

\section{Time to NI values within certain limits}

The mean time till the NI decreased to grade $\mathrm{D}$ was $83.5 \pm 16.4 \mathrm{~s}$ in the TCI-P group compared with $57.3 \pm 18.2 \mathrm{~s}$ in the VCI-S group $(\mathrm{p}<0.001)$. The mean time till the NI level decreased to grade $\mathrm{E}$ and the emergence time in group VCI-S were significantly shorter than those in the TCI-P group $(60.3 \pm 19.2 \mathrm{~s}$ versus $88.0 \pm 17.2 \mathrm{~s}$, respectively, $\mathrm{p}<0.001)$. The mean inspiratory pressure (IP) of mechanical ventilation after anesthesia occurring difference was detected when the patients were turned from the supine to prone position at $5 \mathrm{~min}$ $(\mathrm{p}<0.05)$ (TABLE 2). 


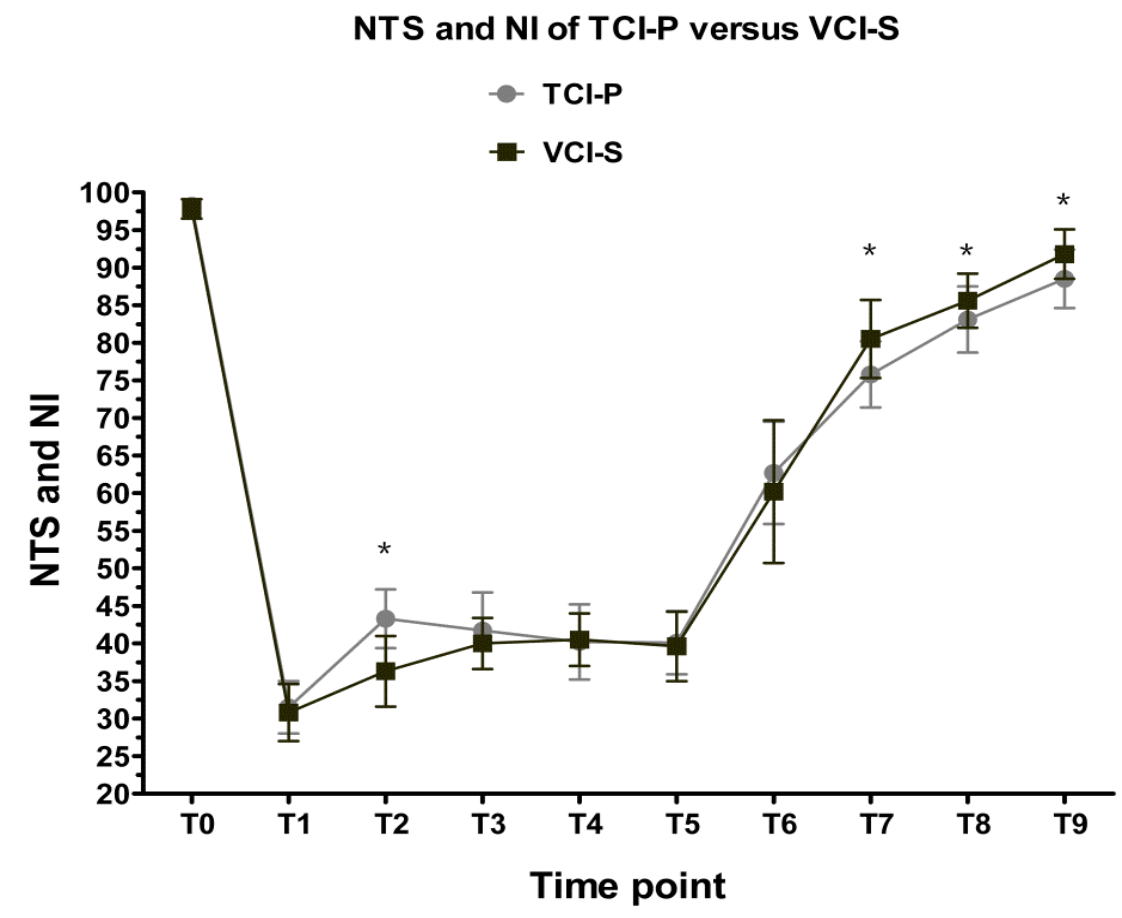

FIGURE 2. Average NI and NTS at different time points during the study period. T0, baseline level before anesthesia; T1, laryngoscopy when NTS level decreased to E stage; T2, T3, after tracheal intubation at 5 minutes, 10 minutes; T4, after patient was turned to the prone position at 5 minutes; T5, during procedure; T6, patient was returned to the supine position after the end of procedure; T7, during the extubation; T8, resumption of spontaneous breathing after the end of procedure; T9, before the patient was transferred to the PACU. NI, narcotrend index; NTS, narcotrend stage; VCI-S, vital capacity inhalation with sevoflurane; $\mathrm{TCI}-\mathrm{P}$, target-controlled infusion induction with propofol. * $\mathrm{P}<0.05$, between groups.

\begin{tabular}{|c|c|c|c|c|}
\hline & $\begin{array}{l}\text { Group TCI-P } \\
\quad(n=30)\end{array}$ & $\begin{array}{l}\text { Group VCI-S } \\
(n=30)\end{array}$ & $\mathrm{T}$ & $P$ \\
\hline NTS at Grade D (s) & $83.5 \pm 16.5$ & $57.3 \pm 18.2^{*}$ & 5.860 & $<0.001$ \\
\hline NTS at Grade E (s) & $88.0 \pm 17.2$ & $60.3 \pm 19.2^{*}$ & 5.896 & $<0.001$ \\
\hline $\begin{array}{l}\text { IP of supine position } \\
(\mathrm{cmH2O})\end{array}$ & $15.5 \pm 1.9$ & $15.1 \pm 1.5$ & 0.998 & 0.323 \\
\hline $\begin{array}{l}\text { IP of Prone position } \\
\left(\mathrm{cmH}_{2} \mathrm{O}\right)\end{array}$ & $16.8 \pm 2.1$ & $15.5 \pm 1.3^{*}$ & 2.950 & 0.005 \\
\hline $\begin{array}{l}\text { IP at } 10 \text { min after prone } \\
\text { position }\left(\mathrm{cmH}_{2} \mathrm{O}\right)\end{array}$ & $17.6 \pm 1.9$ & $16.9 \pm 1.4$ & 1.618 & 0.112 \\
\hline NTS at Grade D (s) & $83.5 \pm 16.5$ & $57.3 \pm 18.2^{*}$ & 5.860 & $<0.001$ \\
\hline
\end{tabular}

\section{- Perioperative hemodynamic stability}

The TCI-P recorded significantly greater in SP after intubation at $5 \mathrm{~min}(\mathrm{P}=0.011)$, in DP after extubation at $10 \mathrm{~min}(\mathrm{P}=0.024)$ (FIGURE 3A and $3 \mathrm{~B})$. The mean HR was also significantly higher in the TCI-P group than that in the VCI-S group from $T_{1}$ to $T_{3}$. However, once the patient returned to the supine position at the end of the procedure the $\left(\mathrm{T}_{6}\right)$, the HR was significantly lower in the TCI-P group than that in the VCI-S group. The HR returned to baseline levels in both groups once the patient was transferred to the PACU (FIGURE 3C).

\section{Consumption of anesthetics agents}

The VCI-S group tended to be more saving than propofol TCI induction during maintenance of anesthesia. There were a statistically significant difference in mean propofol and remifentanil dose between groups for procedures of differing duration, including propofol in preoperative period (TCI-P group: $177.17 \pm 59.01 \mathrm{mg}$; 
A

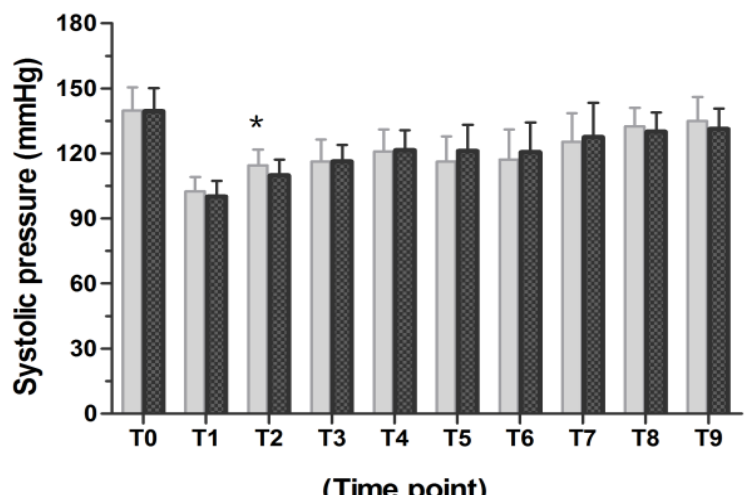

B

Diastolic blood pressure

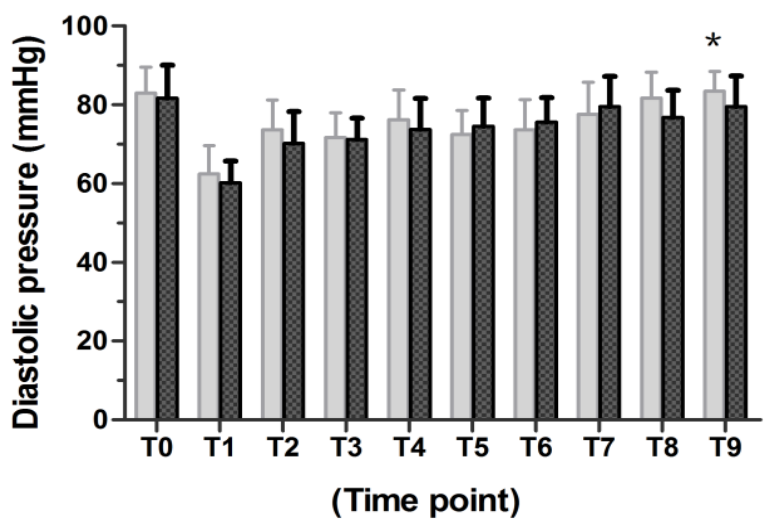

C

Heart rate

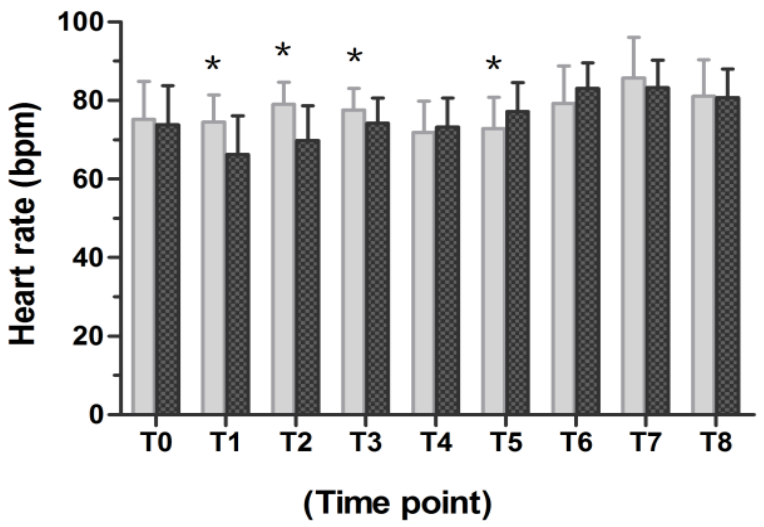

\section{TCI-P VCl-S}

FIGURE 3. Average changes in systolic blood pressure (A), diastolic blood pressure (B) and heart rate (C) at different time points during the study period. T0, baseline level before anesthesia; T1, laryngoscopy when NTS level decreased to E stage; T2, T3, after tracheal intubation at 5 minutes, 10 minutes; T4, after patient was turned to the prone position at 5 minutes; T5, during procedure; T6, patient was returned to the supine position after the end of procedure; T7, during the extubation; T8, resumption of spontaneous breathing after the end of procedure; T9, before the patient was transferred to the PACU. VCI-S, vital capacity inhalation with sevoflurane; TCI-P, target-controlled infusion induction with propofol. *P<0.05, between groups. 
A

Propofol

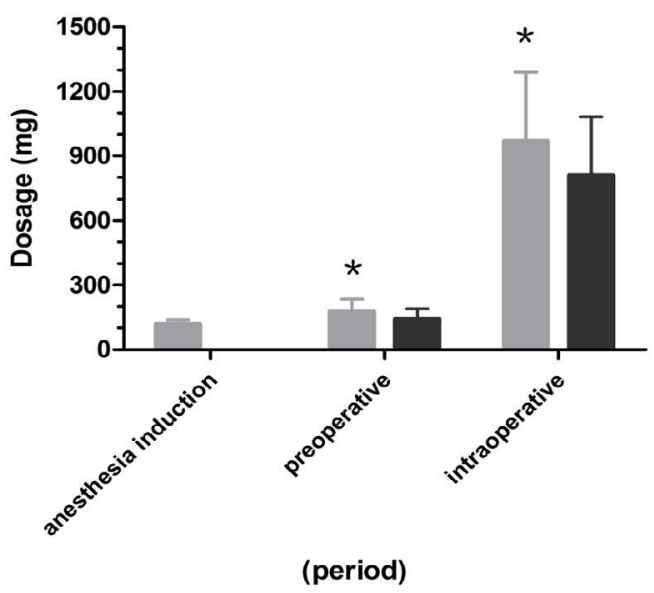

B

\section{Remifentanil}

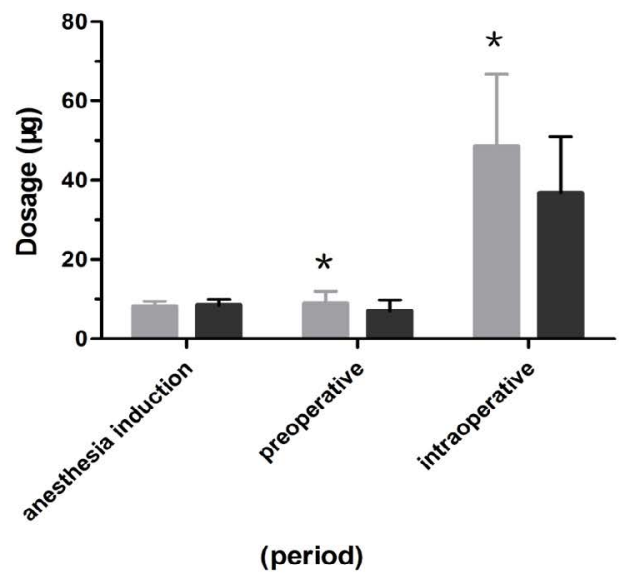

\section{TCI-P VCI-S}

FIGURE 4. The calculated drugs consumption of the maintenance of anesthesia by the two groups in the three study periods. Anesthesia induction, defined as the time from the start of anesthesia induction to tracheal untubation; preoperative, defined as the time from after tracheal intubation to beginning of operation; intraoperative, defined as the time from the start of operation to the end of procedure. ${ }^{*} \mathrm{P}<0.05$, between groups.

VCI-S group: $141.07 \pm 48.03 \mathrm{mg}, \mathrm{P}=0.012)$, in operative period (TCI-P group: $967.50 \pm$ $323.24 \mathrm{mg}$; VCI-S group: $810.10 \pm 272.61 \mathrm{mg}$, $\mathrm{P}=0.046$ ) (FIGURE 4A); and remifentanil in preoperative period (TCI-P group: $8.83 \pm 3.17$ $\mu \mathrm{g}$; VCI-S group: $7.06 \pm 2.72 \mu \mathrm{g}, \mathrm{P}=0.024)$, in operative period (TCI-P group: $48.63 \pm 18.18$ $\mu \mathrm{g}$; VCI-S group: $36.78 \pm 14.22 \mu \mathrm{g}, \mathrm{P}=0.007$ ) (FIGURE 4B).

Overall, the cumulative dosage of remifentanil administered throughout the duration of anesthesia maintenance was significantly reduced in the VCI-S group than in the TCI-P group, $\mathrm{P}=0.008)$. In addition, the VCI-S group required less propofol during anesthetic maintenance than the TCI-P group $(\mathrm{P}=0.038)$ (TABLE 3).

\section{Incidence of adverse effects}

The incidence of adverse effects during the induction and recovery phase of anesthesia were comparable between both groups (TABLE 4).

\section{Discussion}

The question addressed by the present study was whether anesthesia induction with VCI-S in combination with remifentanil would have any major benefit compared with TCI-P combined with remifemtanil for patients undergoing elective lumbar spondylodesis. We found that 


\begin{tabular}{|c|c|c|c|c|}
\hline & Group TCI-P $(n=30)$ & $\begin{array}{c}\text { Group VCI-S } \\
\quad(n=30)\end{array}$ & $\mathrm{T}$ & $P$ \\
\hline Remifentanil dose $(\mu \mathrm{g})$ & $57.5 \pm 21.3$ & $43.8 \pm 16.9^{*}$ & 2.741 & 0.008 \\
\hline Propofol $(\mu \mathrm{g})$ & $1144.7 \pm 382.2$ & $951.2 \pm 320.6^{*}$ & 2.125 & 0.038 \\
\hline Cisatrucroium (mg) & $35.3 \pm 11.1$ & $31.6 \pm 11.5$ & 1.278 & 0.206 \\
\hline \multicolumn{5}{|l|}{ Time of recovery (min) } \\
\hline Respiratory & $8.1 \pm 3.6$ & $7.7 \pm 3.2$ & 0.422 & 0.675 \\
\hline Orientation & $11.8 \pm 5.3$ & $11.1 \pm 6.5$ & 0.433 & 0.667 \\
\hline Extubation & $8.8 \pm 3.7$ & $8.2 \pm 3.9$ & 0.611 & 0.544 \\
\hline
\end{tabular}

SD: standard deviation, $\mathrm{Cl}$ : confidence limits.

Table 4. Adverse effects during induction and recovery of anesthesia. Rate of acceptable induction. Values are number $(\%)[95 \% \mathrm{CI}]$.

\begin{tabular}{|c|c|c|c|c|}
\hline & $\begin{array}{c}\text { Group YCI-P } \\
(n=30)\end{array}$ & $\begin{array}{l}\text { Group VCI-S } \\
\quad(n=30)\end{array}$ & Statistical analysis & $P$ \\
\hline \multicolumn{5}{|c|}{ Anesthesia induction } \\
\hline $\begin{array}{l}\text { Involuntary } \\
\text { movements }\end{array}$ & 3 & 5 & $\begin{array}{c}x^{2}=1.82 \\
\mathrm{df}=1\end{array}$ & 0.178 \\
\hline Coughing & 2 & 4 & $\begin{array}{c}x^{2}=1.80 \\
\mathrm{df}=1\end{array}$ & 0.180 \\
\hline Breath holding & $3(10 \%)$ & $11(37 \%)$ & $\begin{array}{c}x^{2}=15.51 \\
\mathrm{df}=1\end{array}$ & $<0.001$ \\
\hline Laryngospasm & 0 & 1 & $\begin{array}{c}x^{2}=1.01 \\
\mathrm{df}=1\end{array}$ & 0.320 \\
\hline \multicolumn{5}{|c|}{ Anesthesia recovery } \\
\hline Coughing & 4 & 5 & $\begin{array}{c}x^{2}=0.533 \\
\mathrm{df}=1\end{array}$ & 0.465 \\
\hline Laryngospasm & 1 & 1 & $\begin{array}{l}x^{2}=\mathrm{NA} \\
\mathrm{df}=\mathrm{NA}\end{array}$ & NA \\
\hline Restlessness & 7 & 6 & $\begin{array}{c}x^{2}=0.209 \\
\mathrm{df}=1\end{array}$ & 0.647 \\
\hline Total & $20(38 \%)$ & $33(62 \%)$ & $\begin{array}{c}x^{2}=5.760 \\
\mathrm{df}=1\end{array}$ & 0.016 \\
\hline \multicolumn{5}{|l|}{$\begin{array}{c}\text { Acceptability for } \\
\text { anesthesia induction }\end{array}$} \\
\hline Pleasant & 22 & 17 & $\begin{array}{c}x^{2}=1.440 \\
\mathrm{df}=1\end{array}$ & 0.230 \\
\hline $\begin{array}{c}\text { Neither pleasant nor } \\
\text { unpleasant }\end{array}$ & 5 & 7 & $\begin{array}{c}x^{2}=2.560 \\
\mathrm{df}=1\end{array}$ & 0.110 \\
\hline Unpleasant & $3(10 \%)$ & $6(20 \%)$ & $\begin{array}{c}x^{2}=11.56 \\
\mathrm{df}=1\end{array}$ & $<0.001$ \\
\hline \multicolumn{5}{|c|}{ SD: standard deviation, $\mathrm{Cl}$ : confidence limits. } \\
\hline
\end{tabular}

VCI-S used for induction technique on the propofol/remifentanil maintenance regimen was more effective than TCI-P in terms of speed of induction, smooth anesthesia depth, hemodynamic stability, and reduced anesthetic consumption.

In practice, the required depth of anesthesia varies between patients. Anesthesia was uneventful in both the sevoflurane and propofol group, and many standard induction techniques and maintenance regimens have been used with acceptable outcomes. A common combination in clinical practice is to perform induction with propofol and to continue maintenance with sevoflurane $[7,8]$. Compared with propofol, sevoflurane-induced anesthesia has been associated with a faster induction and recovery while offering improved haemodynamic stability 
and an improved cardiovascular patient profile $[9,10]$. However, it is also been associated with a longer duration of convulsion [15]. A vital capacity inhalational induction was used to make the technique akin to that of intravenous bolus injection and there is some evidence that this approach is associated with fewer adverse airway events and is faster than the tidal volume technique $[11,12]$. The present study illustrated that rapid induction and anesthesia depth sufficient for tracheal intubation in vital capacity induction anesthesia can be achieved using a combination of sevoflurane with remifentanil.

In the present study, it is important to understand what was recorded through the NI and NTS and that the interpretation of anesthesia depth could be clearly established. Although the target of the implantation was the relationship between the speed and efficacy throughout the duration of anesthesia induction and not the anesthesia depth, our goal was an NTS between $\mathrm{D}_{0}$ to $\mathrm{E}_{1}$. The time till loss of consciousness as well as the time till the NTS level decreased to stage $\mathrm{E}$ was significantly shorter for patients in the VCI-S group than for those in the TCI-P group. These observations suggest that a high initial concentration of sevoflurane inhalation likely provides a more rapid induction than the target controlled infusion with propofol. In previous studies report that the time to loss of consciousness after induction was significantly longer with sevoflurane when using $2 \%$ initial concentrations than with propofol with a target Cp of $6 \mu \mathrm{g} / \mathrm{ml}$ [13]. Therefore, it is possible that the speed of anesthetic onset is determined in a dose-dependent manner, providing a high aleveolar sevoflurane concentration. We wanted to maintain a clear comparison between the two study groups. All patients in our study received a standard dose of remifentanil during anesthesia induction, where maximizing the speed of reaching a certain depth of anesthesia within which remifentanil could have a hypnotic effect [14]. However, a most studies using BIS monitoring, such as that conducted by Wang et al., have shown that the combination of sevoflurane $(\leq 3.4 \%)$ and remifentanil $(\leq 10$ $\mathrm{ng} / \mathrm{ml}$ ) produced synergistic effects on OAA/S but had no interactive effect on BIS. Wang et al. found that a BIS between 40 and 60 may cause excessive anesthesia when opioids are used to maintain anesthesia [15]. Schmidt et al. [16] have emphasized that only the Narcotrend was able to differentiate between the awake versus steady state anesthesia as well as steady state anesthesia versus first reaction/extubation with a prediction probability value of more than 0.90. Weber et al. [17] found that when the correlation between NI and various nonsteady state end-tidal concentration of the volatile anesthetic sevoflurane (eTS $)$ was examined, the NI was superior to classical EEG parameters as well as hemodynamic parameters in differentiating consciousness from unconsciousness. In our study, although the onset of induction to unconsciousness and NTS stage, ranging from awake to deep hypnosis, were observed, both sevoflurane and propofol caused loss of consciousness and reduction in NI from more than 90 that fell quickly to below 40 and that their effect on NI values is different. The time to reach a certain stage has been shown to be associated with different NTS levels for both drugs, which may be a result of their differing cortical activity effect sensitivities [18]. In addition, the conditions of tracheal intubation were similar in our study, and NI was not significantly difference when NTS was decreased to target limits (stage E) in both groups. A high initial concentration of sevoflurane likely provides a more rapid induction, although the onset and offset of sevoflurane effect as determined by the effect-site elimination rate constant was difficult to determine. Because MAC refers to a state of equilibrium, it was impossible to induce similar sevoflurane concentrations during the inhalation phase and the end-tidal phase during vital capacity induction while maintaining a certain anesthetic depth.

Although induction techaniques is useful in comparing the relative potency between the two groups, making the overall hypnotic control for VCI-S group better than for TCI-P group. However, this difference in control might be attibutable to the pharmacokinetic difference between sevoflurane and propofol combined with remifentanil at the beginning of anesthesia. This difference also probably caused by the multiple confounding factors of anesthetic agents on subcortical structures for the first several minutes after anesthetics induction, launching the patient into a more profound hypnotic trajectory with the sevoflurane of faster onset [13]. Previous studies comparing TCI with propofol and sevoflurane-oxygen-nitrous oxide for anesthesia maintenance in elective spinal surgery of 1-3 h duration have yielded similar findings [19]. Few clinical trials have studied the influence of different anesthetic induction 
strategies on the interaction of anesthetics used during maintenance. For sevoflurane, a significant suppression of flinching behavior and a decrease of fos-like neurons in the dorsal horns of rats, as evaluated by the formalin test, were observed, suggesting that sevoflurane may possess some analgesic properties [20]. Sevoflurane inhalation induced neuronal activation in several different brain regions. Most of the activated neurons were GABAergic, and the number of activated GABAergic neurons increased as the concentration of sevoflurane increased [21]. With respect to remifentanil, Sandeep et al. [22] have studied the interaction between sevoflurane and remifentanil and built response surface pharmacodynanic interaction models using the pooled data for sedation and analgesis endpoints. Clear synergistic interactions were found. Diz and Del Rio [8] found that sevoflurane and propofol, administered together, have an additive interaction in terms of their effect on BIS, but Harris and colleagues [23] demonstrated that this additive interaction, in a simple additive manner, produced loss of consciousness and immobility upon surgical incision, suggesting a common mechanism and/or single site of action. These clinical observations are consistent with a single site of interaction at the gammaaminobutyric acid type A receptor. However, a high initial concentration induction technique with sevoflurane likely offers a similar effect during anesthesia maintenance compared with TCI propofol induction except for a shorter induction time, which may be more common with a TCI propofol in adult patients.

Our results demonstrated no statistical difference in the duration of operation or anesthesia occurring after the induction between the TCI-P and VCI-S groups. However, the total doses of anesthetics (including renmifentanil and propofol) needed for both groups were comparable. Moreover, we did find a significant difference in overall anesthetic consumption between the two groups throughout the duration of anesthesia maintenance. Sevoflurane inhaled induction required fewer doses compared with propofol intravenous induction to achieve a similar anesthesia depth. Previous studies have compared single-agent anesthesia with intravenous propofol (not TCI) and sevoflurane [24,25]. Our study demonstrates that for patients undergoing spinal surgery of up to 3 $\mathrm{h}$ duration, single-agent based anesthesia with TCI propofol or sevoflurane is satisfactory when used together with opiates, as is the standard of care. In the VCI-S group, sevoflurane was used for induction of anesthesia followed by intravenous propofol and remifentanil TCI for maintenance. One problem with this technique is the transition phase from induction to maintenance. An adequate depth of anesthesia is attained with high concentration sevoflurane alone through rapid induction, which can lead to additive interactions with propofol effect during anesthesia maintenance. Several authors have compared the cost of intravenous propofol for the maintenance of anesthesia with different inhalational agents, and the relative cost of propofol exceeds the cost of inhaled anesthetics $[19,26]$. However, the mechanisms that enable a reduced amount of anesthetics consumption during maintenance with propofol-remifentanil in sevoflurane induction are not known. It is our hypothesis that by maximizing the contribution to the anesthetic depth by utilising rapid sevoflurane induction, drugs administered for maintenance that act on different sites of action can, together, provide a synergistic interaction [27].

\section{Strengths and limitations}

The main strength was that the perioperative hemodynamic stability was easily established in our study, as the factors included were classified as ASA I or II and the patients in both groups underwent the same surgical procedure. Most hemodynamic parameters were within acceptable limits when the NTS was kept between stage D and $\mathrm{E}$ of anesthesia maintenance, and no severe hemodynamic changes was recorded in any patient. The introduction of the TCI system has enabled relative accurate dosing by continuous infusion, based on the pharmacokinetic profile of propofol or remifentanil in the average patient [28]. Although one of the advantages of our study is that most of the results were obtained based on NTS or NI, our study did not take into account the amount of anesthetic saved throughout the duration of anesthesia maintenance, which requires an adjustment of the value of the controlled variable according to the per kilogram of body weight in per patient.

However, there are also several limitations that should be acknowledged. The main limitation of our study was that our data was collected from patients undergoing standard anesthetic procedures with drug doses adjusted to the specific needs of the patients, as opposed to standard, controlled dosing regimens determined by experimental conditions 
designed to optimize the pharmacodynamic results [9]. Specifically, our data obtained from the use of the drug Ce values was predicted by a pharmacokinetic model instead of being directly measured in plasma, as has been used in other studies [29]. Another limitation is that anesthesia induction was performed with a predominance of sevoflurane, without achieving steady-state concentrations, and this was combined with TCI remifentanil. This may limit the ability of the anesthetic protocol to predict the depth of anesthesia on NI during induction. Apart from this, fluid administration and blood loss had been identified as one of the factors associated with intraoperative hemodynamic parameters in spinal surgery. Specifically, our data did not include risk factors for such parameters and primarily used the NTS or NI to assess differences in hemodynamic stability.

\section{Conclusion}

We conducted a study on the safety and comparability of anesthesia techniques, including sevoflurane used in induction and propofol used for maintenance of general anesthesia and analysed their effect on NI or NTS. Under the study conditions, it was confirmed that anesthesia induction with vital capacity inhalational sevoflurane resulted in a strong effect on other anesthetics used. This strong effect could produce added utility for the use of perioperative propofol and remifentanil as opposed to intravenous propofol induction, with an anesthesia depth within certain limits using NI or NTS.
Declaration of conflicting interest

The authors declare that there are no conflicts of interest.

\section{Funding}

This research received no specific grant from any funding agency in the public, commercial, or not for-profit sectors.

\section{Authors' contributions}

Chunshan Dong, MD, PhD: have made substantial contributions to conception and design, or analysis and interpretation of data.

Peng Sun, MD: have been involved in drafting the manuscript or revising it critically for important intellectual content.

Junma Yu, MD: agree to be accountable for all aspects of the work in ensuring that questions related to the accuracy or integrity of any part of the work are appropriately investigated and resolved.

Jun Zhang, MD: acquisition of data

Chao Wu, BD: acquisition of data

\section{Acknowledgements}

We gratefully acknowledge the assistance of the behalf of AstraZeneca and ShangHai hengrui pharmaceuticals Ltd regarding references provide, and Ms Fan luyan, Pharmaceutical department, third affiliated hospital of Anhui Medical University for help with pharmacology analysis. 


\section{References}

Hendrickx JFA, Eger EI, Sonner JM, Shafer SL. Is synergy the rule? A review of anesthetic interactions producing hypnosis and immobility. Anesth. Analg. 107(2), 494-506 (2008).

Rohm KD, Piper SN, Suttner S, Schuler $S$, Boldt J. Early recovery, cognitive function and costs of a desflurane inhalational vs. a total intravenous anaesthesia regimen in long-term surgery. Acta Anesthesiol. Scand. 50(1), 14-18 (2006).

Sneyd JR, Andrews CJH, Tsubokawa T. Comparison of propofol/remifentanil and sevoflurane/remifentanil for maintenance of anaesthesia for elective intracranial surgery. Br. J. Anaesth. 94(6), 296-302 (2005).

Visser K, Hassink EA, Bonsel GJ, Moen J, Kalkman CJ. Randomized controlled trial of total intravenous anaesthesia with propofol versus inhalational anaesthesia with isoflurane-nitrous oxide. Anesthesiol. 95(3), 616-626 (2001)

EI-Radaideh KM, AJ-Ghazo MA. Single breath vital capacity induction of anesthesia with $8 \%$ sevoflurane versus intravenous propofol for laryngeal tube insertion in adults. Saudi Med. J. 28(1), 36-40 (2007).

Troy AM, Huthinson RC, Rasy WR, Kenney GN. Tracheal intubating conditions using propofol and remifentanil target-controlled infusion. Anaesthesia. 50(12), 12041207 (2002).

Schumacher PM, Dossche J, Mortier EP, et al. Response surface modeling of the interaction between propofol and sevoflurane. Anesthesiol. 111(4), 790804 (2009).

Diz JC, Del Rio R, Lamas A, et al. Analysis of pharmacodynamic interaction of sevoflurane and propofol on bispectral index during general anaesthesia using a response surface model. $\mathrm{Br}$. J. Anaesth. 104(6), 733-739 (2010).

Liao R, Li JY, Liu GY. Comparison of sevoflurane volatile induction/ maintenance anaesthesia and propofol/ remifentanil total intravenous anaesthesia for rigid bronchoscopy under spontaneous breathing for tracheal/bronchial foreign body removal in children. Eur. J. Anaesthesiol. 27(11), 930-934 (2010).

Filipovic M, Michaux I, Wang J, et al. Effects of sevoflurane and propofol on left ventricular diastolic function in patients with preexisting diastolic dysfunction. Br. J. Anaesth. 98(1), 1218 (2007).

Yurino M, Kimura H. A comparison of vital capacity breath and tidal breathing techniques for induction of anesthesia with high sevoflurane concentration in nitrous oxide and oxygen. Anaesthesia. 50(4), 308-311 (1995).

Ruffle JM, Snider MT. Comparison of rapid and conventional inhalation induction of halothane oxygen anesthesia in healthy men and women. Anesthesiol. 67(4), 584-587 (1987).

Velly LJ, Rey MF, Bruder NJ, et al. Differential dynamic of action on cortical and subcortical structures if anesthetic agents during induction of anesthesia. Anesthesiol. 107(2), 202212 (2007).

Koitabashi T, Johansen JW, Sebel PS. Remifentanil dose/ electroencephalogram bispectral response during combined propofol/ regional anesthesia. Anesth. Analg. 94(6), 1530-1533 (2002).

Wang HL, Yang L, Guo XY, et al. Response surface analysis of sevoflurane -remifentanil interactions on consciousness during anesthesia. Chin. Med. J. 125(15), 2682-2687 (2012).

Schmidt GN, Bischoff P, Standi T, et al. Narcotrend and Bispectral index monitor are superior to classic electroencephalographic parameters for the assessment of anesthetic states during propofol-remifentanil anesthesia. Anesthesiol. 99(5), 10721077 (2003).

Weber F, Hollnberger H, Gruber M, Frank B, Taeger K. The correlation of the Narcotrend Index with endtidal sevoflurane concentration and hemodynamic parameters in children. Paediatr. Anaesth. 15(9), 727-732 (2005).

Jacob Z, Li H, Makaryus $\mathrm{R}$, et al. Metabolomic profiling of children's brains undergoing general anesthesia with sevoflurane and propofol. Anesthesiol. 117(5), 1062-1071 (2012).

Watson KR, Shah MV. Clinical comparison of 'single agent' anesthesia with sevflurane versus target controlled infusion of propofol. Br. J. Anaesth. 85(4), 541-546 (2000).

Hao S, Takahata O, Mamiya K, Iwasaki $\mathrm{H}$. Saveoflurane suppresses noxious stimulus-evoked expression of Foslike immunoreactivity in the rat spinal cord via activation of endogenous opioid systems. Life Sci. 71(5), 571580 (2002).

Han LC, Zhang H, Wang W, et al. The effect of sevoflurane inhalation on gabaergic neurons activation: observation on the GABA67-GFP knock-in mouse. Anat. Rec. (Hoboken) 293(12), 2114-2122 (2010).

Manyam SC, Gupta DK, Johnson KB, et al. Opioid-volatile anesthetic synergy: a response surface model with remifentanil and sevoflurane as prototype. Anesthesiol. 105(2), 267278 (2006).

Harris RS, Lazar O, Johansen JW, Sebel PS. Interaction of propofol and sevoflurane on loss of consciousness and movement to skin incision during general anesthesia. Anesthesiol. 104(6), 1170-1175 (2006).

Jellish WS, Lien CA, Fontenot HJ, Hall R. The comparative effects of sevoflurane versus propofol in the induction and maintenance of anesthesia in adult patients. Anesth. Analg. 82(3), 479485 (1996).

Thwaite A, Edmends S, Smith I. Inhalation induction with sevoflurane: a doubleblind comparison with propofol. $\mathrm{Br}$. J. Anaesth. 78(4), 356-361 (1997).

Stevanovic PD, Petrova G, Miljkovic $\mathrm{B}$, et al. Low fresh gas flow balanced 
anesthesia versus target controlled intravenous infusion anesthesia in laparoscopic cholecystectomy: a costminimization analysis. Clin. Ther. 30(9), 1714-1725 (2008).

Shafer SL, Hendrickx JFA, Flood P, Sonner J, Eger B. Additivity versus synergy: a theoretical analysis of implications for anesthetic mechanisms. Anesth. Analg. 107(2), 507-524 (2008).

Reader J. Opioid or propofol: what kind of drug for what kind of sedation? Manual dosing or target-controlled infusion? Anesth. Analg. 108(3), 704-
706 (2009).

Jeleazcov C, Ihmsen H, Schmidt J, et al. Pharmacodynamic modelling of the bispectral index response to propofolbased anesthesia during general surgery in children. Br. J. Anaesth. 100(4), 509-516 (2008).

This special issue on Current Trends in Clinical Research was edited by Dr. Mohamed Elsayed.

This special issue on Current Trends in Clinical Research was edited by Dr. Mohamed Elsayed. 